\title{
ON QUASI-CONVEX FUNCTIONS AND RELATED TOPICS
}

\author{
KHALIDA INAYAT NOOR \\ Department of Mathematics \\ Girls college for Science Education \\ Sitteen Road, Al-Malaz, Riyadh \\ Saudi Arabia
}

(Received January 28, 1985 and in revised form March 20, 1985)

ABSTRACT. Let $S$ be the class of functions $f$ which are analytic and univalent in the unit disc $E$ with $f(0)=0, f^{\prime}(0)=1$. Let $C, s^{*}$ and $K$ be the classes of convex, starlike and close-to-convex functions respectively. The class $C^{\star}$ of quasiconvex functions is defined as follows:

Let $f$ be analytic in $E$ and $f(0)=0, f^{\prime}(0)=1$. Then $f \varepsilon C^{\star}$ if and only if there exists a $g \in C$ such that, for $\approx \mathrm{E}$

$$
\operatorname{Re} \frac{\left(z f^{\prime}(z)\right)^{\prime}}{g^{\prime}(z)}>0
$$

In this paper, an up-to-date complete study of the class $c^{\star}$ is given. Its basic properties, its relationship with other subclasses of $S$, coefficient problems, arc length problem and many other results are included in this study. Some related classes are also defined and studied in some detail.

IEY WORDS AND PHRASES. Univalent convex, alpha-convex, quasi-convex, alpha quasiconvex, close-to-convex, arclength, coefficient, radius of convexity, order $\beta$ type $Y$, Livingston's and Libera's operators.

1980 AMS SUBJBCT CLASSIFICATION CODB. 30A32, 30A34.

\section{INTRODUCTION.}

Denote by $S$ the class of functions $f$ which are analytic and univalent in the unit disc $E$ and satisfy $f(0)=0, f^{\prime}(0)=1$. The subclasses $s^{*}$ and $C$ of starlike and convex functions respectively are well-known and have been extensively studied, see [1], [2] and [3]. A function $f$ is said to be in $s^{*}$ if and only if for $\quad z E \mathrm{E}$

$$
\operatorname{Re} \frac{z f^{\prime}(z)}{f(z)}>0
$$

$S^{*}$ and $C$ are related by the Alexander relation [4], that is

$$
\text { fєC if, and only if } z f^{\prime} \varepsilon S^{*}
$$


Hence a function $f$ is said to be in $C$, if and only if for $z \varepsilon E$

$$
\operatorname{Re} \frac{\left(z f^{\prime}(z)\right)^{\prime}}{f^{\prime}(z)}>0
$$

The subclass $K$ of $S$ consisting of close-to-convex functions is also we11 known [5] and many properties of $S^{*}$ can be extended to the wider class $K$. A function $f$ is said to be in $K$ if and only if there exists a convex function $g$ such that, for $z \varepsilon \mathrm{E}$

$$
\operatorname{Re} \frac{f^{\prime}(z)}{g^{\prime}(z)}>0
$$

Since $G=z g^{\prime}$ is starlike for $g$ convex, (1.4) can be written as

$$
\operatorname{Re} \frac{z f^{\prime}(z)}{G(z)}>0
$$

for $z \in E$ and $G \in S^{*}$. Taking $G(z)=f(z)$ in $(1.5)$ one sees that $S^{*} \subset K$, which shows that

$$
\mathrm{C} \subset \mathrm{S}^{*} \subset \mathrm{K}
$$

\section{QUASI-CONVEX FUNCTIONS.}

We proceed now to define and discuss a subclass of $S$ which is related to $K$ by an Alexander type relation [6].

DEFInITIOn 2.1. Let $f$ be analytic in $E$ with $f(0)=0, f^{\prime}(0)=1$. Then $f$ is said to be quasi-convex in $E$ if and only if there exists a convex function $g$ with $g(0)=0, g^{\prime}(0)=1$ such that, for $z \in E$

$$
\operatorname{Re} \frac{\left(z f^{\prime}(z)\right)^{\prime}}{g^{\prime}(z)}>0
$$

Denote the class of quasi-convex functions by $c^{*}$.

It is clear that, when $f(z)=g(z)$ and $g \varepsilon C$, then $(2.1)$ holds. Hence $C \subset C^{*}$. We show now that $C^{\star} \subset K$, so that every quasi-convex function is univalent.

THEOREM 2.1. Let $f \in C^{*}$. Then, for $z \in E$,

$$
\operatorname{Re} \frac{z f^{\prime}(z)}{g(z)}>0, \quad g \varepsilon C
$$

and so $c^{\star} \subset K \subset S$, thus, every quas1-convex function is close-to-convex and hence univalent in $\mathrm{E}$. 
PROOF. A result of Libera [7] shows that, if $s$ and $t$ are functions analytic in $E$ with $s(0)=t(0)=0$ and $t \varepsilon S^{\star}$, then for $z \varepsilon E$,

$$
\operatorname{Re} \frac{s^{\prime}(z)}{t^{\prime}(z)}>0 \rightarrow \operatorname{Re} \frac{s(z)}{t(z)}>0
$$

An Immediate application of this with $s(z)=z f^{\prime}(z)$ and $t(z)=g(z)$ proves the theorem. It follows at once from the definition (2.1) that

$$
f \varepsilon C^{*} \quad \text { if and only } \text { if } z f^{\prime} \varepsilon K
$$

We now extend some results to the class $C^{*}$ which are known to be true for $C$, see [1], [2] and [3].

THBorm 2.2. Let $f \in C^{\star}$ with $f(z)=z+\sum_{n=2}^{\infty} a_{n} z^{n}$. Then, for $|z|=r<1$,

$$
\begin{aligned}
& \text { (1) }\left|a_{n}\right|<1, \quad n=2,3, \ldots \\
& \text { (11) } \frac{1}{(1+r)^{2}}<\left|f^{\prime}(z)\right|<\frac{1}{(1-r)^{2}} \\
& \text { (111) } \frac{r}{1+r}<|f(z)|<\frac{r}{1-r} \\
& \text { (iv) }|\omega|>\frac{1}{2} \text {, where } f(z) \neq \omega \text { in E. }
\end{aligned}
$$

All inequalities are sharp, equality belng attalned for

$$
f_{0}(z)=\frac{z}{1-z}
$$

Pro0r. (1) Since $f \in C^{*}$, there exists a convex function $g$ with $g(z)=$ $z+\sum_{n=2}^{\infty} b_{n} z^{n}$, such that, for $z \in E$

$$
\frac{\left(z f^{\prime}(z)\right)^{\prime}}{g^{\prime}(z)}=h(z), \text { where } \operatorname{Re} h(z)>0,
$$

and $h(z)=\sum_{n=0}^{\infty} c_{n} z^{n}, \quad c_{0}=1$

So

$$
\left(z f^{\prime}(z)\right)^{\prime}=g^{\prime}(z) h(z)
$$

Equating the coefficient of $z^{n-1}$ on both sides, we have

$$
n^{2} a_{n}=n b_{n}+(n-1) c_{1} b_{n-1}+(n-2) c_{2} b_{n-2}+\ldots+2 c_{n-2} b_{2}+c_{n-1}
$$

1.e.

$$
n^{2}\left|a_{n}\right|<n\left|b_{n}\right|+(n-1)\left|c_{1}\right|\left|b_{n-1}\right|+\ldots \ldots+2\left|c_{n-2}\right|\left|b_{2}\right|+\left|c_{n-1}\right| \cdot
$$


Now, from the known results [7], $\left|b_{n}\right|<1, n=2,3, \ldots$, and $\left|c_{n}\right|<2, n=1,2,3 \ldots$, we have

$$
n^{2}\left|a_{n}\right|<n+\frac{2\{n(n-1)\}}{2}=n^{2}, \quad n=2,3, \ldots
$$

and this implies $\left|a_{n}\right|<1, n=2,3, \ldots$

$$
\left|\left(z f^{\prime}(z)\right)^{\prime}\right|=\left|g^{\prime}(z) h(z)\right|
$$

Using known [1,2 and 3] distortion theorems for the functions $g$ and $h$, we have

$$
\frac{1-r}{(1+r)^{3}}<\left|\left(z f^{\prime}(z)\right) \cdot\right|<\frac{1+r}{(1-r)^{3}}
$$

Integrating the right hand side of (2.5) from 0 to $z$, we obtain

$$
\begin{aligned}
\left|z f^{\prime}(z)\right| & <\int_{0}^{z}\left|\left(z f^{\prime}(z)\right) \cdot\right| d z \\
& <\int_{0}^{r} \frac{1+r}{(1-r)^{3}} d r=\frac{r}{(1-r)^{2}}
\end{aligned}
$$

In order to obtain a lower bound for $\left|f^{\prime}(z)\right|$, we proceed as follows. Let d be the radius of the open disc contained in the map of $E$ by $z f^{\prime}$. Let $z_{0}$ be the point of $|z|=r$ for which $\left|z f^{\prime}(z)\right|$ assumes its minimum value. This minimum increases with $\left\{r\right.$ the image of $|z|<r$ by $w=f^{\prime}(z)$ expands $\}$ and is less than $d_{1}$. Hence the linear-segment connecting the origin with the point $z_{0} f^{\prime}\left(z_{0}\right)$ will be covered entirely by the values of $z f^{\prime}(z)$ in $E$. Let $\ell$ be the arc in $E$ which is mapped by wezf'(z) onto this 1inear-segment. Then

$$
\begin{aligned}
\left|z f^{\prime}(z)\right| & =\int_{l}\left|\left(z f^{\prime}(z)\right)^{\prime}\right| d|z| \\
& >\int \frac{1-r}{(1+r)^{3}} d r \\
& =\frac{r}{(1+r)^{2}}
\end{aligned}
$$

Integrating (ii), we obtain (iii) and by letting $r \rightarrow 1$ in the lefthand side of (iii), we have (iv).

Waadeland [8] proved that every starlike m-fold symmetric function $g$, with $g(z)=z+\sum_{k=1}^{\infty} b_{m k+1} z^{m k+1}$ satisfies

$$
\begin{aligned}
\left|b_{m k+1}\right| & <\left(\begin{array}{l}
\frac{2}{m}+k-1 \\
k
\end{array}\right) \\
& \simeq \frac{1}{m \Gamma\left(\frac{2}{m}\right)} k^{\frac{2}{m}-2}
\end{aligned}
$$


In order to extend this result to $C^{*}$, we need only to extend Waadeland's result to $K$ and then use the relationship between $C^{*}$ and $K$. However this extension to $K$ was done by Pommerenke [9] and so (2.6) is true for $f \varepsilon \mathrm{C}^{\star}$.

The following result for the class $c^{\star}$ follows exactly in the same way as for the class $C$ in $[10]$.

THEORBM 2.3. Let $f \varepsilon c^{*}$ with $f(z)=z+\sum_{n=2}^{\infty} a_{n} z^{n}$, and $g(z)=z+\sum_{k=2}^{\infty} b_{k} z^{k}$.

Let $g(z) \propto f(z)$. Then, for all $n$,

$$
s_{n}\left(\frac{1}{2} z\right) \propto f(z)
$$

where

$$
\begin{aligned}
S_{n}(z)=z+\sum_{k=2}^{n} b_{k} z^{k} & \\
& (\propto \text { means "subordinate to") }
\end{aligned}
$$

Clunie and Keogh [10] showed that if fEC with $f(z)=z+\sum_{n=2}^{\infty} a_{n} z^{n}$ and $f(E)$ has definite area then $n a_{n}=o(1)$ as $n+\infty$. This result has been extended to $c^{*}$ in [6] as follows.

THBOREM 2.4. Let $f \in C^{\star}$ with $f(z)=z+\sum_{n=2}^{\infty} a_{n} z^{n}$. If $f(E)$ has finite area, then $n a_{n}=o(1)$ as $n+\infty$, the index of $n$ being best possible.

Denote by $C(r)$ the closed curve which is the image of $f\left(E_{r}\right)$ and by $L(r)$ the length of $\mathrm{C}(\mathrm{r})$. We prove:

THEOma $2.5[6]$. Let $\mathrm{f}^{*} \mathrm{C}^{*}$. Then, for $0<\mathrm{r}<1$,

$$
2 \sqrt{(\pi A(r))}<L(r)<2 \sqrt{(\pi A(\sqrt{r}))}\left(\log \frac{1}{1-r}\right) 1 / 2
$$

Further, if $A(r)<\infty$ for $0<r<1$, then

$$
L(r)=o(1)\left(\log \frac{1}{1-r}\right) \quad \text { as } \quad r+1
$$

The convex function $f(z)=\log \frac{1}{1-r}$ shows that the factor $\left(\log \frac{1}{1-r}\right)^{1 / 2}$ in $(2.7)$ is the best possible.

PRO0F. The left hand inequality follows at once from the isoperimetric inequality. Since $f \varepsilon C^{*}, F(z)=z f^{\prime}(z)$ is close-to-convex. Thus

$$
\begin{aligned}
L(r) & =\int_{0}^{2 \pi}\left|z f^{\prime}(z)\right| d \theta=\int_{0}^{2 \pi}|F(z)| d \theta \\
& <2 \pi \int_{0}^{r} M\left(\rho, z f^{\prime}\right) \frac{d \rho}{\rho}, \text { see }[1, p .45]
\end{aligned}
$$




$$
\begin{aligned}
& <2 \pi \sum_{n=1}^{\infty}\left|a_{n}\right| r^{n} \\
& \left.<2 \pi \sum_{n=1}^{\infty} n\left|a_{n}\right|^{2} r^{n}\right)^{1 / 2}\left(\sum_{n=1}^{\infty} \frac{r^{n}}{n}\right)^{1 / 2} \\
& =2 \sqrt{(\pi A(\sqrt{r}))} \quad\left(\log \frac{1}{1-r}\right)^{1 / 2}
\end{aligned}
$$

We can show (2.8) easily by taking $A(r)<\infty$.

REMARR 2.1. For $f \varepsilon C$, it is wel1-known that $L(r)<2 \pi M(r)$. It follows from (2.7) that for $f \in C^{*}, L(r)=O(1) M(r)\left(\log \frac{1}{1-r}\right)^{1 / 2}$ as $r \rightarrow 1$. The question of whether the factor $\left(\log \frac{1}{1-r}\right)^{1 / 2}$ can be removed is sti11 open.

It is we11-known [11] that

$$
\operatorname{Re} \frac{\left(z f^{\prime}(z)\right)^{\prime}}{f^{\prime}(z)}>0+\operatorname{Re} \frac{z f^{\prime}(z)}{f(z)}>\frac{1}{2}, \quad z \varepsilon E,
$$

that 1s, every convex function is starlike of order $\frac{1}{2}$. It is natural to ask if such a relationship exists between $\mathrm{C}^{*}$ and $\mathrm{K}$. The following example shows that this is not in fact the case. Then

$$
\text { EXAMLE 2.1. Take } f(z)=z, g(z)=\frac{z}{1-\alpha z}, \frac{1}{2}<\alpha<\frac{1}{\sqrt{2}} \text {. }
$$

$$
\operatorname{Re} \frac{\left(z f^{\prime}(z)\right)^{\prime}}{g^{\prime}(z)}=\operatorname{Re}(1-\alpha z)^{2}>0, \quad z \varepsilon E,
$$

but

$$
\operatorname{Re} \frac{z f^{\prime}(z)}{g(z)}=\operatorname{Re}(1-\alpha z),
$$

and

$$
\text { Inf } \operatorname{Re} \frac{z f^{\prime}(z)}{g(z)}<\frac{1}{2} \text { for } \frac{1}{2}<\alpha<\frac{1}{\sqrt{2}}, \quad z \in E .
$$

Now, following the same method as in [12], we have

THBORKM 2.6. Let $f \varepsilon c^{*}$ and $g(z)=\frac{f^{\prime}(z)}{f^{\prime}(-z)}$. Then $\operatorname{Re}(g(z))^{1 / 3}>0$, for $z \varepsilon E$. This result is sharp as can be seen from the function

$$
z f_{1}^{\prime}(z)=[z(1-\mu z)] /\left[(1+z)^{2}\right]
$$

where $\mu=(\cos \gamma) e^{i \gamma}, 0<\gamma<\pi$, and $f_{1} \varepsilon c^{*}$ with respect to the convex function $\phi(z)=\frac{\left[-1 e^{1 \gamma} z\right]}{(1+z)}$. 3. RELATIONSHIP OP $c^{\star}$ MITH OTHER SUBCLASSES OF $s$.

(1) The class $C$. The class $c$ of convex functions is a proper subclass of $c^{\star}$. In fact if we write 


$$
F(z)=\frac{f[(x+z) /(1+\bar{x} z)]-f(x)}{f^{\prime}(x)\left(1-|x|^{2}\right)}, \quad x \in E, \quad z \varepsilon E
$$

where

$$
f(z)=\frac{z}{(1-z)^{2}}
$$

then the function $f_{*}(z)$ defined in $E$ by

$$
\mathbf{f}_{\star}(z)=\int_{0}^{z} \frac{F(\xi)}{\xi} d \xi
$$

belongs to $C^{*}$ but not to $C$, see [6] for more details.

(11) The Class $\mathrm{s}^{*}$.

The class $c^{\star}$, while a proper subclass of the class $k$ of close-to-convex functions, is not contained in $\mathrm{s}^{*}$. For example, the function

$$
f(z)=\frac{1-1}{2} \frac{z}{1-z}-\frac{1+1}{2} \log (1-z)
$$

belong to $c^{*}$ but for sufficiently small $\varepsilon, \operatorname{Re} \frac{z f^{\prime}(z)}{f(z)}<0$, when $z=e^{1 \theta},-\varepsilon<\theta<0$. This means $f$ is not star11ke. Also the Koebe function $k(z)=\frac{z}{(1-z)^{2}}$ is starlike but does not belong to $c^{*}$. It is clear from the coefficient result and the distortion theorems for the class $\mathrm{C}^{*}$.

(111) The Class $R_{\alpha}$.

The class $R_{\alpha}$ of univalent functions was introduced by Reade [13] and studied by Pommerenke [9]. We define $R_{\alpha}$ as follows:

An anlytic function $f$ with $f^{\prime}(z) \neq 0$ belongs to $R_{\alpha}, 0<\alpha<1$, If and only if

$$
\int_{\theta}^{\theta} \operatorname{Re}\left\{\frac{\left(z f^{\prime}(z)\right)^{\prime}}{f^{\prime}(z)}\right\} d \theta>-\alpha \pi
$$

for all $\theta_{1}, \theta_{2}$ such that $0<\theta_{1}<\theta_{2}<2 \pi, 0<\mathrm{r}<1$.

Before establishing a relationship between $C^{*}$ and $R_{\alpha}$, we need the following necessary condition for $C^{*}$.

THBOREM 3.1. Let $f \varepsilon \mathrm{C}^{*}$ and $z=\mathrm{re}^{1 \theta}, 0<\mathrm{r}<1$. Then, for $0<\theta_{1}<\theta_{2}<2 \pi$,

$$
\int_{\theta}^{\theta} \operatorname{Re}\left\{\frac{\left(z f^{\prime}(z)\right)^{\prime}}{f^{\prime}(z)}\right\} d \theta>-\pi+\frac{\theta^{-\theta_{1}}}{2}
$$

PRO0F. It has been proved in [14] that for $z f^{\prime}=F \varepsilon K$, and for all $\theta_{1}, \theta_{2}$ such that $0<\theta_{1}<\theta_{2}<2 \pi$

$$
-\pi+\frac{\theta_{2}-\theta}{2}<\int_{\theta}^{\theta} \operatorname{Re}\left\{\frac{r e^{1 \theta} F^{\prime}\left(\mathrm{re}^{1 \theta}\right)}{\mathrm{F}\left(\mathrm{re}^{1 \theta}\right)}\right\} \mathrm{d} \theta<2 \pi+\frac{{ }^{\theta_{2}-\theta} 1}{2}
$$


using this and the fact that $f \varepsilon C^{\star}$ if and only if $z f^{\prime} \varepsilon K$, we obtain the required result.

We note that in $(3.1), \frac{\theta_{2}^{-\theta} 1}{2}$ can be very small and we can take $\frac{\theta_{2}^{-\theta} 1}{2}=\varepsilon \pi$, where $0<\varepsilon<1$. Thus we conclude that

for some $\alpha,(0<\alpha<1)$.

$$
C^{*} \subset R_{\alpha}
$$

REMARK 3.1. It is an open problem to find the exact value of $\alpha \varepsilon(0,1)$ that goes with $\mathrm{C}^{*}$. It should be some fixed number determined by $\mathrm{C}^{*}$.

(iv) The class of functions convex in one direction.

Robertson [15] introduced the class $c_{1}$ of convex functions in one direction. These are the functions for which the intersection of the image region with each line of certain fixed direction is either empty or one interval. He has also shown that if $f$ has real coefficients, then $f \in C_{1}$ if and on $l y$ if $z f^{\prime} \varepsilon T$, where $T$ is the class of typically real functions, that is, the functions with real coefficients.

We prove the following:

THEOREM 3.2. If $f \varepsilon \mathrm{C}^{\star}$ in $\mathrm{E}$ and has real coefficients, then it is convex in one direction.

PROOF. Let $C^{*}(R), K(R)$ and $C_{1}(R)$ be the classes of functions which are in $C^{\star}, K$ and $C_{1}$ respectively and have real coefficients. Let $f \varepsilon C^{\star}(R)$. This implies $z f^{\prime} \varepsilon K(R)$. But $K(R) \subset T$. Hence $z f^{\prime} \varepsilon T$ and so $f \varepsilon C_{1}(R)$. Hence $C^{\star}(R) \subset C_{1}(R)$ and this proves the theorem.

From Theorem 3.2 and the results for the class $C_{1}(R)$ in [15], we have:

THEOREA 3.3. Let $\mathrm{f} \varepsilon \mathrm{C}^{\star}(\mathrm{R})$. Then

(1) $\quad \operatorname{Re} \frac{f(z)}{z}>\frac{1}{2}$ and $\frac{f(z)}{z}$ is subordinate to $(1+z)^{-1}$.

(ii) $\frac{1+\left|a_{2}\right| r}{1+2\left|a_{2}\right| r+r^{2}}<\operatorname{Re}\left\{\frac{f\left(r e^{i \theta}\right)}{r e^{i \theta}}\right\}<\frac{1+\left|a_{2}\right| r}{1-r^{2}}$,

where $\quad f(z)=z+\sum_{n=2}^{\infty} a_{n} z^{n}$

(iii) $L(r)<\frac{2 \pi r}{\left(1-r^{2}\right)}$, where $L(r)$ is the length of the closed curve $f\left(E_{r}\right)$. The equality is obtained for $f(z)=\frac{z}{1-z}$.

(iv) $\left|\arg \frac{f(z)}{z}\right|<\arcsin |z|$

and

, $\approx \mathrm{E}$

$\left|\arg f^{\prime}(z)\right|<2 \arcsin |z|$

(v) $F \varepsilon C^{*}(R)$, where 


$$
F(z)=\int_{0}^{1} f(t z) d \phi(t)=z+\sum_{n=2}^{\infty} \mu_{n} a_{n} z^{n},
$$

$\phi(t)$ is any real function monotonic increasing in the interval $(0,1)$ and the moments sequence $\left\{\mu_{n}\right\}$ is given by

$$
\mu_{n}=\int_{0}^{1} t^{n} d \phi(t), \mu_{1}=1
$$

Thus we have seen that

(1) $\quad C \subset C^{*} \subset R_{\alpha} \subset K \subset S$

(2) $\quad C^{*}(R) \subset C(R) \subset T$

We now discuss the relationship of $c^{*}$ with other subclasses in a different way. We have the following:

THBORBy 3.4. Let $f \varepsilon c^{*}$ in $E$. Then $f$ maps $|z|<r=4 \sqrt{2}-5 \simeq 0.6568$ onto a convex domain, and this result is sharp.

This follows at once from the result of Lewandowsk1 [16] where he proved that the exact radius $r_{1}$ such that the image of $|z|<r_{1}$ by $f \varepsilon K$ is a starshaped domain (with respect to the origin) is

$$
r_{1}=4 \sqrt{2}-5 \simeq 0.6568
$$

We see that, from this result $f \varepsilon C^{*} \rightarrow z f^{\prime} \varepsilon K \rightarrow z f^{\prime} \varepsilon S^{*}$ for $|z|<4 \sqrt{2}-5 \rightarrow$ f $\varepsilon C$ for $|z|<4 \sqrt{2}-5$

Lewandowsk1's method ylelds the existence of an extremal function which maps $E$ onto the w-plane cut along a half-line not passing through the origin consequently we have the extremal function for theorem 3.4 .

THEOREM 3.5. Let $f \varepsilon \mathrm{C}^{*}$ and $\mathrm{g} \varepsilon \mathrm{C}$ in E. If $\operatorname{Re} \frac{z f^{\prime}(z)}{g(z)}>0, z \varepsilon E$, then $\operatorname{Re} \frac{\left(z f^{\prime}(z)\right)^{\prime}}{g^{\prime}(z)}>0$, for $|z|<\frac{1}{3}$ For the proof, see [17].

4. APPLICATIONS OF THE GLASS $c^{\star}$.

(a) The Class $\mathrm{K}_{1}$.

We now introduce a new class $K_{1}$ by replacing convex function $g$ in (1.4) with quasi-convex function. This generalizes the concept of qasi-convexity and close-toconvexity both.

DEFIKITION 4.1. Let $f$ be analytic in $E$ and $f(0)=0, f^{\prime}(0)=1$. Then $f \varepsilon K_{1}$, If and only if, there exists a $g \varepsilon C^{*}$, such that for $z \varepsilon E$,

$$
\operatorname{Re} \frac{f^{\prime}(z)}{g^{\prime}(z)}>0
$$

Clearly $\mathrm{C}^{\star} \subset \mathrm{K} \subset \mathrm{K}_{1}$.

We state some basic properties of the class $\mathrm{K}_{1}$. We refer to [18] for the proofs. 
THEOREM 4.1. Let $f \varepsilon K_{1}$ and be given by $f(z)=z+\sum_{n=2}^{\infty} a_{n} z^{n}$. Then

(1) $\left|a_{n}\right|<n, \quad$ for all $n$.

(11) $\quad \frac{(1-r)}{(1+r)^{3}}<\left|f^{\prime}(z)\right|<\frac{(1+r)}{(1-r)^{3}}$$$
\frac{r}{(1+r)^{2}}<|f(z)|<\frac{r}{(1-r)^{2}}
$$

(1v) $\quad|w|>\frac{1}{4}$, where $f(z) \neq w$ in $E$.

All Inequalities are sharp, equality being attalned for

$$
\begin{aligned}
& f_{0}(z)=\frac{z}{(1-z)^{2}} \varepsilon K_{1} \\
& \text { (v) } \quad\left\|a_{n+1}|-| a_{n}\right\|=o(1) \text {, for all } n,
\end{aligned}
$$

where $O(1)$ denotes a constant.

$$
\text { (vi) } \quad L(r)=o(1)\left[A(\sqrt{r}) \frac{1}{1-r}\right]^{1 / 2}, \quad 0<r<1
$$

The question whether the factor $\left(\frac{1}{1-r}\right)^{1 / 2}$ can be Improved is unsettled and remains open.

$$
\text { (v11) For } f \in K_{1} \text { Implies that } z f^{\prime} \text { is univalent in }|z|<\frac{1}{5} \text {. }
$$

(b) Alpha-quasi-convex functions.

Mocanu [19] Introduced the class $M_{\alpha}$ of alpha-convex functions as follows:

Let $\alpha$ be real and suppose that $f: f(z)=z+\sum_{n=2}^{\infty} a_{n} z^{n}$ is analytic in $E$ with $f(z) . f^{\prime}(z) \neq 0$. Then $f \varepsilon M_{\alpha}$ if, for $z \varepsilon E$,

$$
\operatorname{Re}\left\{(1-\alpha) \frac{z f^{\prime}(z)}{f(z)}+\alpha \frac{\left(z f^{\prime}(z)\right)^{\prime}}{f^{\prime}(z)}\right\}>0
$$

It has been shown [20] that all $\alpha$-convex functions are unfvalent and starlike and they unify the classes of starlike $(\alpha=0)$ and convex $(\alpha=1)$ functions.

Using the concept of quasi-convexity, we now define the following:

DBFINITION 4.2. Let $\alpha$ be real and $f: f(z)=z+\sum_{n=2}^{\infty} a_{n} z^{n}$ be analyt1c in E. Then $f$ is sald to be alpha-quasi-convex, if and only if there exists a convex function g such that, for $z \varepsilon E$ 


$$
\operatorname{Re}\left\{(1-\alpha) \frac{f^{\prime}(z)}{g^{\prime}(z)}+\alpha \frac{\left(z f^{\prime}(z)\right)}{g^{\prime}(z)}\right\}>0
$$

We denote the class of $\alpha$-quasi-convex functions as $Q_{\alpha}$. We note that $Q_{0}=K$ and $Q_{1}=C^{*}$. Thus alpha-quasi-convex functions connect the classes $K$ and $c^{*}$ in the same way as alpha-convex functions do $s^{*}$ and $C$.

In [21], we proved:

\section{THBOREM 4.2.}

(1) Let $F(z)=(1-\alpha) f(z)+\alpha z f^{\prime}(z)$, and $\alpha$ be rea1, $\alpha>0, z \varepsilon E$. Then $f \varepsilon Q_{\alpha}$, 1f and only if, $F \in K$.

(11) $f \varepsilon Q_{\alpha}$, if and only 1f, for $\alpha>0$, there exists a close-to-convex function $F$ such that, for $z \varepsilon E$

$$
f(z)=\frac{1}{\alpha} z^{1-\frac{1}{\alpha}} \int_{0}^{z} t^{-2+\frac{1}{\alpha}} F(t) d t
$$

(111) Every $\alpha$-quasi-convex function, for $0<\alpha<1$ is close-to-convex.

(iv) Let $F \in K$ in $E$. Then $F$ w111 be $\alpha$-quas1-convex in $|z|<r_{0}=1 /\left(2 \alpha+\sqrt{\left.\left(4 \alpha^{2}-2 \alpha+1\right)\right)}\right.$. This result is sharp.

(v) Let $f \varepsilon Q_{\alpha}$ and be given by $f(z)=z+\sum_{n=2}^{\infty} a_{n} z^{n}$. Then, for $n>2$,

$$
\left|a_{n}\right|<\frac{n}{1+\alpha(n-1)}
$$

This result is sharp as can be seen from the function

$$
f_{0}(z)=\frac{1}{\alpha} z^{1-\frac{1}{\alpha}} \int_{0}^{z} t^{\frac{1}{\alpha}-1}(1-t)^{-2} d t
$$

(c) The class $C^{*}(\beta, \gamma)$ of quasi-convex functions of order $\beta$ type $\gamma$.

A function $f \varepsilon S$ is called a convex function of order $\beta, 0<\beta<1$ if, for $z \varepsilon E$,

$$
\operatorname{Re} \frac{\left(z f^{\prime}(z)\right)^{\prime}}{f^{\prime}(z)}>B
$$

We note this class by $C(B)$.

Also $f \in S$ is a starlike function of order $B, 0<\beta<1$, for $z \varepsilon E$

$$
\operatorname{Re} \frac{z f^{\prime}(z)}{f(z)}>B
$$

and we call this class as $s^{\star}(\beta)$. These two classes were introduced by Robertson [22]. In [23], Libera introduced the close-to-convex functions of order $\beta$ type $\gamma$. 
DEPINITION 4.3. A function $f$ analytic in $E$, normalized by the conditions $f(0)=0, f^{\prime}(0)=1$, is sald to be close-to-convex of order $B$ type $\gamma$ where $0<\beta<1$ and $0<\gamma<1$, if and only if there exists a function $g \varepsilon S^{\star}(\gamma)$ such that, for $z \varepsilon E$

$$
\operatorname{Re} \frac{z f^{\prime}(z)}{g(z)}>B
$$

We denote such a class of functions as $K(\beta, \gamma)$. It is clear that $K(0,0)=K$.

We now introduce terminology of order and type together in the class $C^{*}$ as:

DEFINITION 4.4. A function $f$, analytic in $E$, normalized by the conditions $f(0)=0, f^{\prime}(0)=1$, is said to be quas 1 -convex of order $\beta$ type $\gamma$, if and only if there exists a function $g \in C(\gamma)$ such that for $z \in E$

$$
\operatorname{Re} \frac{\left(z f^{\prime}(z)\right)^{\prime}}{g^{\prime}(z)}>B
$$

where $0<\beta<1$ and $0<\gamma<1$. We call such a class as $C^{*}(\beta, \gamma)$. Clearly $C^{*}(0,0)=C^{*}$.

We shall now state some results on the class $C^{*}(\beta, \gamma)$. For the proofs, we refer to $[24]$.

THEOREM 4.3. Every quasi-convex function of order $\beta$ type $\gamma$ is close-to-convex of the same order and hence univalent.

REMAR 4.1. From the definition of $C^{*}(\beta, \gamma)$, we can see that an Alexander-type relation holds between the classes $C^{\star}(\beta, \gamma)$ and $K(\beta, \gamma)$, that is

$$
f \in C^{*}(\beta, \gamma) \text { if and only if } \quad \mathrm{f}^{\prime} \varepsilon \mathrm{K}(\beta, \gamma)
$$

THBORBM 4.4. Let $f \varepsilon C^{*}(\beta, \gamma)$ and be given by $f(z)=z+\sum_{n=2}^{\infty} a_{n} z^{n}$. Then we have (1) $\left|a_{n}\right|<\frac{2(3-\gamma) \ldots \ldots \ldots(n-2 \gamma)[n(1-\beta)+(\beta-\gamma)]}{n_{0} n !}$

(11) $\frac{1}{r} \int_{0}^{r} \frac{(1-r) d r}{(1+r)^{2-2 \gamma}[1+(1-2 \beta) r]}<\left|f^{\prime}(z)\right|$

$$
\left\{\begin{array}{c}
r(1-\gamma)(1-2 \beta)+(\beta-\gamma)\left[1-(1-r)^{2-2 \gamma}\right] \\
\gamma \neq 2, \gamma \neq 1 \\
(1-2 \beta) \frac{1}{r} \log (1-r)+\frac{2(1-\beta)}{r}, \gamma=\frac{1}{2} \\
2(\beta-1) \frac{1}{r} \log (1-r)+(2 \beta-1), \gamma=1,
\end{array}\right.
$$

where $|z|=r, 0<r<1$. The first result and right-hand side of the second are sharp. THEOREM 4.5. Let $\mathrm{f} \varepsilon \mathrm{S}$ and $\mathrm{g} \varepsilon \mathrm{C}$. Let $\operatorname{Re} \frac{z \mathrm{f}^{\prime}(z)}{\mathrm{g}(\mathrm{z})}>\beta$, for $z \varepsilon \mathrm{E}$. Then $\mathrm{f} \varepsilon \mathrm{C}^{\star}(\beta, 0)$ for $|z|<r_{0}=\frac{1}{3}$, and $f \varepsilon C^{*}$ for $|z|<r_{1}=\frac{1}{3-4 B}$. 
We can also define the class $Q(\alpha, \beta, \gamma)$ of alpha-quasi-convex functions of order $\beta$ type $\gamma$ as:

DEFINITION 4.6. Let $\alpha>0$. A function $f$, analytic in $E$, is said to be alphaquasi-convex of order $\beta$ type $\gamma$, if and only if there exists a function $g \in C(\gamma)$ such that

$$
\operatorname{Re}\left[(1-\alpha) \frac{f^{\prime}(z)}{g^{\prime}(z)}+\alpha \frac{\left(z f^{\prime}(z)\right)^{\prime}}{g^{\prime}(z)}\right]>B
$$

for $z E \mathrm{E}$, and $\beta, \gamma \in[0,1]$. We denote this class of functions as $Q(\alpha, \beta, \gamma)$. It is clear that $Q(\alpha, 0,0)=Q_{\alpha}$. For different values of $\alpha, \beta$, and $\gamma$, we have

$$
\begin{array}{ll}
Q(0, \beta, \gamma)=K(\beta, \gamma) & Q(0,0,0)=K \\
Q(1, \beta, \gamma)=C^{*}(\beta, \gamma), & Q(1,0,0)=c^{*}
\end{array}
$$

We notice that this class unifies the two classes $K(\beta, \gamma)$ and $C^{\star}(\beta, \gamma)$, and it follows from the definition that

$$
f \varepsilon Q(\alpha, \beta, \gamma) \text { if and only if }\left\{(1-\alpha) f+\alpha z f^{\prime}\right\} \varepsilon K(\beta, \gamma)
$$

Integral representation and coefficient problem can be solved in the same way as we did for the class $Q_{\alpha}$.

(d) OPBrators ON THE Classes $C^{*}(\beta, \gamma)$.

Let $f=T(F)$, where $T$ is an operator. Now we shall be dealing with the mapping properties of $f$ when $F \varepsilon C^{\star}(\beta, \gamma), \beta, \gamma \varepsilon[0,1]$ and $T$ is a differential or integral operator.

He re we shall discuss the case when $T$ is an integral operator $I$. In [7], Libera considered the operator $\mathrm{I}: \mathrm{H}_{0} \rightarrow \mathrm{H}_{0}$,

$$
H_{0}(E)=\left\{f \mid f(z)=z+\sum_{n=2}^{\infty} a_{n} z^{n} \text { and analytic in } E\right.
$$

where for $F \varepsilon \mathrm{H}_{0}, I(F)=f$ and

$$
f(z)=\frac{2}{z} \int_{0}^{z} F(t) d t
$$

He proved that

$$
\left.\begin{array}{l}
I(C) \subset c \\
I\left(s^{*}\right) \subset s^{*} \\
I(K) \subset K
\end{array}\right\}
$$


A generalization of (4.2) has been considered in [25] by taking operator $I_{n}$ defined as $I_{n}: H_{0}+H_{0}, I_{n}(F)=f$ and

$$
f(z)=n z^{-n+1} \int_{0}^{z} t^{n-2} F(t) d t, n=1,2,3, \ldots
$$

A simple proof of (4.2) is given by Mocanu, see [19], where it is also shown that

$$
\left.\begin{array}{l}
I\left(s^{*}\right) \subset s^{*}(\gamma) \\
I(C) \subset c(\gamma)
\end{array}\right\}
$$

where $\gamma=(-3+\sqrt{17)} / 4$ and is the same in both expressions.

Pascu [26] considered the operator $I_{\lambda}, 0<\lambda<1, I: H_{0} \rightarrow H_{0}, I_{\lambda}(F)=f$,

$$
f(z)=\frac{1}{\lambda} z^{1-\frac{1}{\lambda}} \int_{0}^{z} z^{\frac{1}{\lambda}-2} F(z) d z
$$

which generalizes the results in (4.2) and (4.4).

In [27], Salagean studied the operators (4.5) for the classes $S^{*}(\gamma)$ and $C(\gamma)$. By using the same techniques used in [26] we obtain the similar results for the classes $K(B, \gamma)$ and thus consequently we have:

THBORBY 4.6. Let $0<\lambda<1$. Let $\mathrm{f}$ be defined by $(4.5)$ where $F \varepsilon C^{\star}(\beta, \gamma), \beta, \gamma \in[0.1]$. Then $f \in C^{*}(\mu, \sigma)$ where $\beta<\mu<1$ and $\sigma$ is defined as follows:

(1) If $0<\lambda<\frac{1}{2}$ and $\frac{\lambda}{2(\lambda-1)}<\gamma<1$, then

$$
\sigma=\sigma_{1}=\left[2 \lambda \gamma+\lambda-2+\sqrt{4 \lambda^{2} \gamma^{2}-12 \lambda^{2} \gamma+8 \lambda \gamma+9 \lambda^{2}-4 \lambda+4}\right] / 4 \lambda>0
$$

and

(11) If $\frac{1}{2}<\lambda<1$ and $\frac{\lambda-1}{2 \lambda}<\frac{3 \lambda-\sqrt{8 \lambda}}{2 \lambda}<\gamma$, then

$$
\sigma=\sigma_{2}=\left[2 \lambda \gamma+\lambda-\sqrt{4 \lambda^{2} \gamma^{2}-12 \lambda^{2} \gamma+9 \lambda^{2}-8 \lambda}\right] / 4 \lambda>0
$$

and

(111) If $\frac{1}{2}<\lambda<1$ and $\frac{\lambda-1}{2 \lambda}<\frac{3 \lambda-\sqrt{8 \lambda}}{2 \lambda}<\gamma<1$, then $\sigma=\sigma_{1}$

Spectal Cases:

(1) For $\lambda=\frac{1}{2}, \gamma=0, \beta=0$, we obtain a known result for the class $C^{*}$, see [16].

(11) For $\beta=0, \gamma=0$, we see that

$$
\mathrm{F} \varepsilon \mathrm{C}^{*}+\mathrm{f} \varepsilon \mathrm{C}^{*}
$$


THEOREM 4.7. Let $0<\lambda<1$ and $0<\beta<1$. Let $f$ be given by (4.5) and $F \varepsilon Q(\alpha, \beta, \gamma)$ where $0<\gamma<1, \alpha>0$. Then $f \varepsilon Q(\alpha, \beta, \gamma)$.

For the proofs of the above results, we refer to [28]. Special Cases:

(1) For $\lambda=\frac{1}{2}, \gamma=0, \beta=0$, we obtain this result for the class $Q_{\alpha}$, see [21].

(1i) For $\gamma=0, \beta=0$, we see that

$$
\mathrm{F} \varepsilon Q_{\alpha} \rightarrow \mathrm{f} \varepsilon Q_{\alpha}
$$

REMARK 4.2. Using the integral representation of $f \in Q(\alpha, \beta, \gamma)$ and theorem 4.7, we notice that, for $0<\alpha<1$

$$
Q(\alpha, \beta, \gamma) \subset K(\beta, \gamma)
$$

In [29], Livingston has studied the converse question considered by Libera [7]. In fact he studied the mapping properties of the function $f$ defined by

$$
f(z)=D(F(z))=\frac{1}{2}(z F(z))^{\prime},
$$

where $D$ is a differential operator and $F$ is one of the subclasses of S. For example he has proved that if $\mathrm{FES}^{*}$, then $\mathrm{f}$, given by (4.6), is starlike for $|z|<\frac{1}{2}$ and, in general, in no larger disc centered at the origin.

Padmanabhan [30] has refined the results of Livingston by imposing further restrictions on the character of $F$. His main theorem shows that $1 f \mathrm{~F} \in \mathrm{S}^{\star}(\gamma)$, for $0<\gamma<\frac{1}{2}$, then $f$, defined by $(4.6)$, is starlike of the same order for $\left.|z|<\{\gamma-2)+\left(\gamma^{2}+4\right)^{1 / 2}\right\} / 2 \gamma$. He obtains analogous result when $F$ is a convex function of order $\gamma$. Libera and Livingston [31] extended and generalized the results of Padmanabhan in the following ways. They extended to include the range of $\gamma$ when $\frac{1}{2} \gamma \gamma<1$ and generalized by finding, the sharp radius of the disc in which $\left\{\operatorname{Re} \frac{z f^{\prime}(z)}{f(z)}>\sigma\right\}$ when $\mathrm{FES}^{*}(\gamma), 0<\gamma<1,0<\sigma<1$ and $\sigma>\gamma$. They were not able to obtain suitable results for the complimentary case when $\sigma<\gamma$, but in [32] Bajpal and Singh gave a method which covers both of the cases and their result is the best possible.

We can generalize the Livingston differential operator $D$ as following:

$$
D_{\lambda}(f)=f(z)=(1-\lambda) F(z)+\lambda z F^{\prime}(z),
$$

where $\lambda>0$ and $z \in E$. The mapping properties of the function $f$, when $F$ is in one of the subclasses of $S$ have been studied in [33].

We generalize Libera and Livingston's result by replacing Livingston's operator (4.6) by the operators (4.7) and have the following:

THBOREM 4.8. Let $0<\beta<1,0<\gamma<1, \gamma<\sigma<1$ and $\beta<\mu<1$. Let $F \varepsilon C^{*}(\beta, \gamma)$ and $f$ be

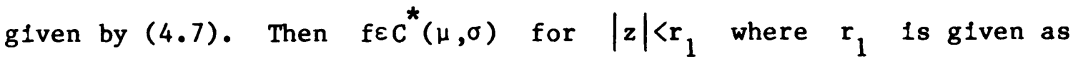




$$
r_{1}=\min \left(r_{0}, r_{2}\right)
$$

where $r_{0}$ is the smallest positive root of

$$
(1-\sigma)+2\{(\gamma-\sigma)+\lambda(1-\gamma)(\sigma-2)\} r+(2 \gamma-\sigma-1)(1-2 \lambda(1-\gamma)) r^{2}=0,
$$

and $r_{2}$ is the smallest positive root of the equation

$$
\begin{aligned}
{[1-(1-2 \lambda(1-\gamma)) r][(1-\mu)} & +2\{(\beta-\mu)+\lambda(\beta+\gamma+\mu(1-\gamma)-2)\} \mathbf{r} \\
+ & \left.(2 \beta-\mu-1)(1-2 \lambda(1-\gamma)) r^{2}\right]=0
\end{aligned}
$$

For $\beta=\gamma=0$, this result reduces to one for the class $C^{*}$, see [34].

THBOREM 4.9. Let $\alpha>0$ and $\lambda>0$. Let $0<\beta, \gamma<1, \beta<\mu<1$ and $\gamma<\sigma<1$. If $F \varepsilon Q(\alpha, \beta . \gamma)$ and $f$ is $g$ iven by $(4.7)$, then $f \varepsilon Q(\alpha, \mu, \sigma)$ for $|z|<r_{1}$ where $r_{1}$ is defined as in theroem 4.8 .

When $\beta=\gamma=0$, we obtain this result for the class $Q_{\alpha}$, see [34]. For the proofs of the above theorems we refer to [35].

We can demonstrate the relationship between all the subclasses of $S$ as follows:

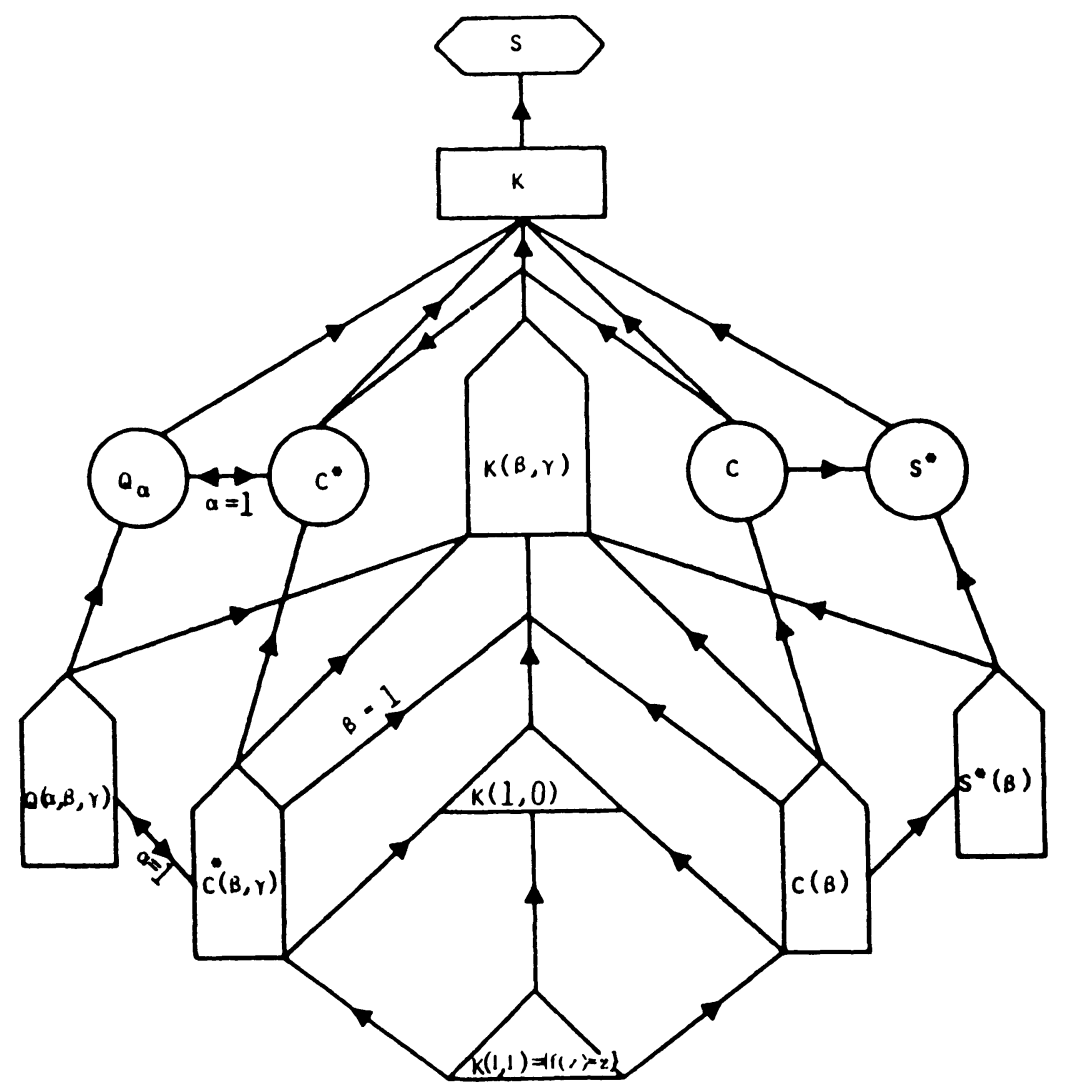

$E:\{z:|z|<1\}, \longrightarrow$ : Set Inclusion, $0<\alpha<1$, and $\beta, \gamma \in[0,1]$ 


\section{REFERENCES}

1. HAYMaN, W. K. Multivalent Functions, Cambridge University Press, U.K. 1967.

2. DUREN, P. L. Univalent Functions, Springer-Verlag, Berlin, 1983.

3. GoOden, A. W. Univalent Functions, Vo1. I, II, Mariner, Tampa, FL, 1983.

4. ALEXANDER, J. W. Functions Which Map the Interior of the Unit Circle Upon Simple Regions, Ann. Math. 17 1915-16, 12-22.

5. KAPLAN, W. Close-to-convex Schlicht Functions, Mich. Math. J. 1 1952, 169-185.

6. MOOR, K. I. and THOMAS, D.R. Quasi-convex Univalent Functions, Int. J. Math. \& Math. Sci. $31980,255-266$.

7. LIBERA, R. J. Some Classes of Regular Univalent Functions, Proc. Amer. Math. Soc. $161965,755-758$.

8. WAADELAND, H. Uber k-fold Symmetrische Sternformige Schlichte Abbildungen des Einheitskreises, Math. Scand. 3 1955, 150-154.

9. POMMRREKE, Ch. On the Coefficients of Close-to-convex Functions, Mich. Math. J. ㅇ $1962,259-269$.

10. CHUNIE, J. G. and KRIGH, F. R. On Starlike and Convex Schlicht Functions, J. London Math. Soc. $351960,229-235$.

11. STrohiacker, E. Beitrage zur Theorie der Schtichten Funktionen, Math. Zeit. 37 $1933,356-380$.

12. BURDIC, G. R., KEOGH, F. R. and MERKES, E.P. On a Ratio of Univalent Functions, J. Math. Ana 1. App1. 53 1974, 221-224.

13. READE, M. O. The Coefficients of Close-to-convex Functions, Duke Math. J. 23 $1956,459-462$.

14. KIM, Y. J. and MERKS, B. P. On Certain Convex Sets in the Space of Locally Schlicht Functions, Trans. Amer. Math. Soc. 196 1974, 217-224.

15. ROBERTSOA, M. S. Analytic Functions Starlike in One Di rection, Amer. J. Math. 58 $1936,465-472$.

16. NOOR, K. I. On a Subclass of Close-to-convex Functions, Comm. Math. Univ. St. Pauli 29 1980, 25-28.

17. NOOR, K. I. Some Radius of Convexity Problems, C.R. Math. Rep. Acad. Sci. Canada 5 $1982,283-286$.

18. NOOR, K. I. and AL-OBOUDI, F. M. A Generalized Class of Close-to-convex Functions, Pak. J. Sci. Res. 341982 .

19. Mocaru, P. T. Une Propriete de Convexite Generalizee Dams la Theorie de la Representation Conforms, Math. (Cluj) (34), 11 1969, 127-133.

20. MILler, S. S., Mocanu, P. and READE, M. Al $1 \alpha$-convex Functions a re Univalent and Starlike, Proc. Ame r. Math. Soc. 2 1973, 553-554.

21. NOOR, R. I. and AL-OBOUDI, F. M. Alpha-quasi-convex Functions, Car. J. Math. $\underline{3}(1984), 1-8$.

22. ROBERTSON, M. S. On the Theory of Univalent Functions, Ann. Math. 37 1936, 374-408.

23. LIBERA, R. J. Some Radius of Convexity Problems, Duke Math. J. 1964, 143-158.

24. NOOR, $K_{0}$ I. The Class $C^{*}(\beta, \gamma)$ of Quasi-convex Functions of Order Type, to appear.

25. BerNard, S. M. Convex and Starlike Univalent Functions, Trans. Amer. Math. Soc. $1351969,429-446$.

26. PASC, N. N. Alpha-starlike Convex Functions, to appear.

27. SALAGEAN, G. S. Properties of Starlikeness and Convexity by Some Integral Operators, Lect. Notes Math. №. 743, Springer-Verlag, Berlin, 367-372.

28. NOOR, R. I. and AI-RHORASANI, H. A. Properties of Close-to-convexity Preserved by Some Integral Operators, J. Math. Anal. Appl., 112 (1985), 509-516. 
29. LIVIngston, A. B. On the Radius of Univalence of Certain Analytic Functions, Proc. Amer. Math. Soc. $171966,352-357$.

30. PADMANABHAN, K. S. On the Radius of Unfvalence of Certain Classes of Analytic Functions, J. London Math. Soc. (2) 1 1969, 225-231.

31. LIBERA, R. J. and LIVINGSTON, A. B. On the Univalence of Some Classes of Regular Functions, Proc. Amer. Math. Soc. 30 1971, 327-336.

32. BAJPI, P. L. and SINGH, P. The Radius of Starlikeness of Certain Analytic Functions, Proc. Amer. Math. Soc. 44 1974, 395-402.

33. NOOR, K. I., AL-OBOUDI, F.M. and AL-DIHAN, N. A. On the Radius of Univalence of Convex Combinations of Analytic Functions, Int. J. Math. \& Math. Sc1. 6 $1983,335-340$.

34. NOOR, R. I. and AL-DIHAN, N. A. A Subclass of Close-to-convex Functions, Punjab Univ. J. Math. 14-15 1981-82, 183-192.

35. NOOR, R. I. and AL-KHORASANI, H. A. Generalization of Livingston's Operator for Certain Classes of Unfvalent Functions, to appear. 


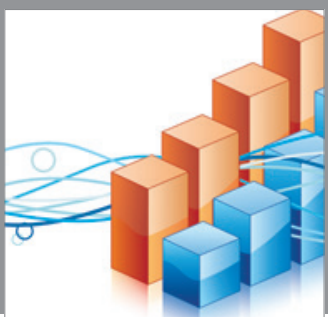

Advances in

Operations Research

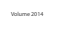

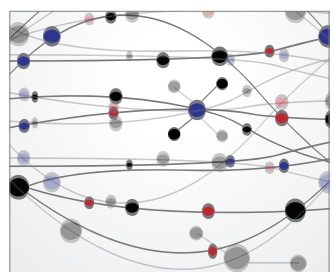

\section{The Scientific} World Journal
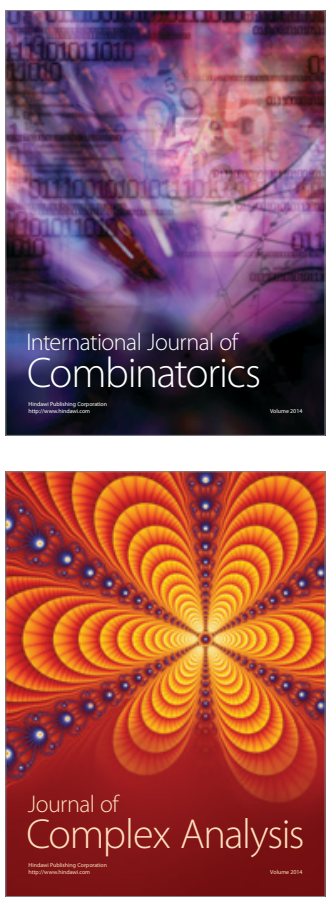

International Journal of

Mathematics and

Mathematical

Sciences
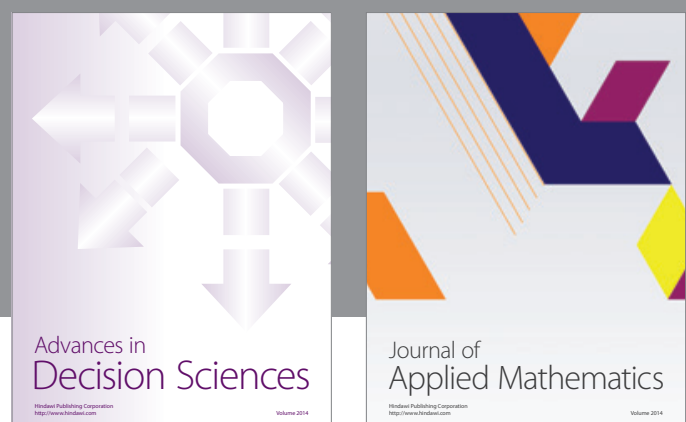

Journal of

Applied Mathematics
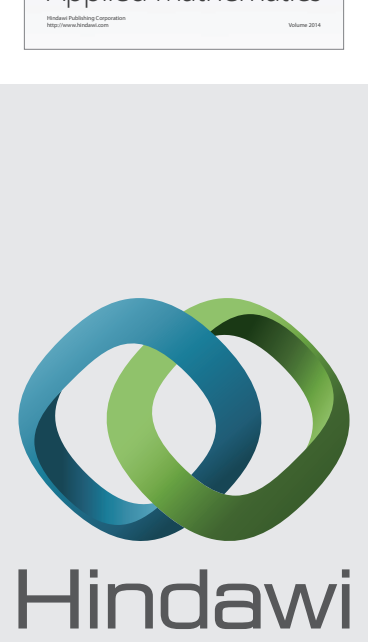

Submit your manuscripts at http://www.hindawi.com
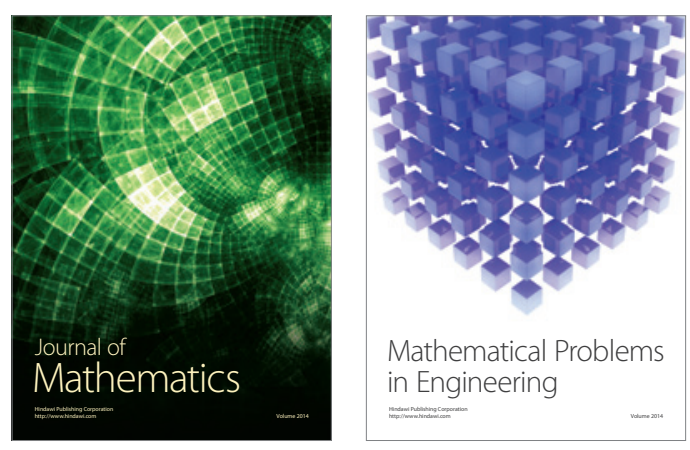

Mathematical Problems in Engineering
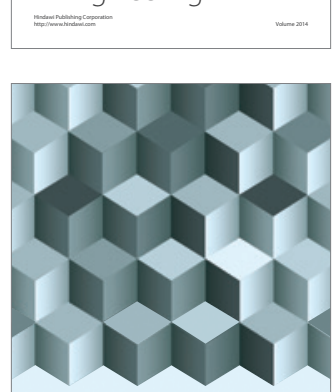

Journal of

Function Spaces
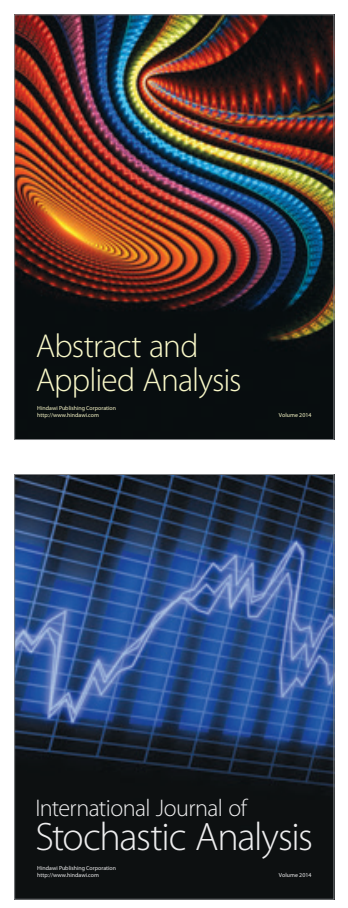

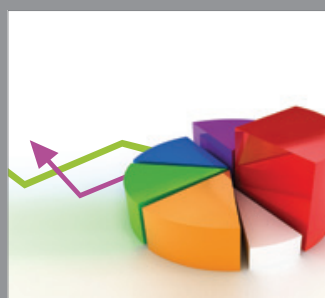

ournal of

Probability and Statistics

Promensencen
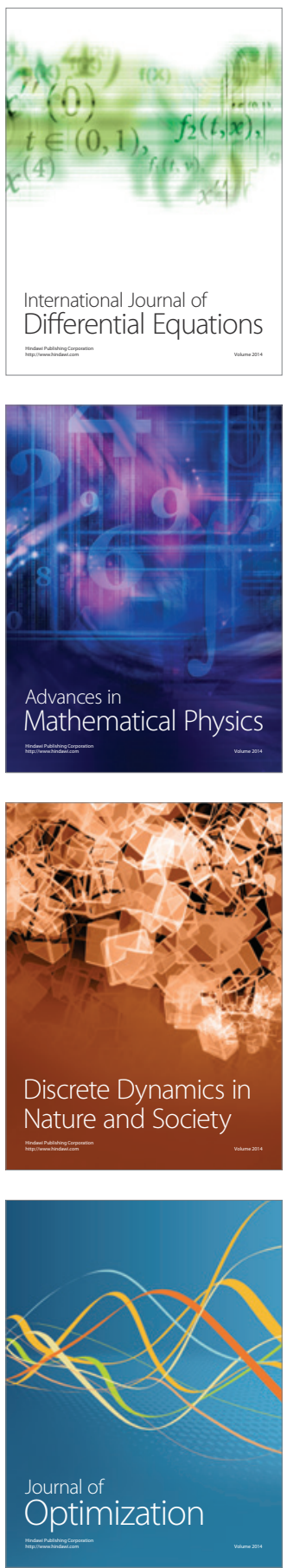